\title{
THE METHOD OF UPPER, LOWER SOLUTIONS AND MONOTONE ITERATIVE SCHEME FOR HIGHER ORDER HYPERBOLIC PARTIAL DIFFERENTIAL EQUATIONS
}

\author{
RAVI P. AGARWAL \\ (Received 25 September 1987) \\ Communicated by A. J. Pryde
}

\begin{abstract}
Uniformly monotone convergent iterative methods for obtaining multiple solutions of $(n+m)$ th order hyperbolic partial differential equations together with initial conditions are discussed. Appropriate partial differential inequalities which connect upper and lower solutions, and variation of parameters formula is developed.
\end{abstract}

1980 Mathematics subject classification (Amer. Math. Soc.) (1985 Revision): 35 H 05, 35 A 35.

\section{Introduction}

For $a, b \in \mathbf{R}, a>0, b>0$, let $I_{a}, I_{b}$ denote the intervals $[0, a]$ and $[0, b]$ respectively, and $I_{a b}$ the rectangle $I_{a} \times I_{b}$. On $I_{a b}$ we shall consider the following nonlinear hyperbolic partial differential equation

$$
\frac{\partial^{n+m} u}{\partial x^{n} \partial y^{m}}=f(x, y,\langle u\rangle)
$$

together with the initial conditions

$$
\begin{aligned}
\frac{\partial^{j} u(x, 0)}{\partial y^{j}} & =\alpha_{j}(x), & & 0 \leq j \leq m-1, \\
\frac{\partial^{i} u(0, y)}{\partial x^{i}} & =\beta_{i}(y), & & 0 \leq i \leq n-1,
\end{aligned}
$$

(C) 1989 Australian Mathematical Society $0263-6115 / 89 \$ A 2.00+0.00$ 
where $\langle u\rangle$ stands for

$$
\begin{aligned}
\left(u, \frac{\partial u}{\partial x}, \ldots, \frac{\partial^{n} u}{\partial x^{n}}, \frac{\partial u}{\partial y}, \ldots, \frac{\partial^{n+1} u}{\partial x^{n} \partial y}, \ldots, \frac{\partial^{m-1} u}{\partial y^{m-1}}, \ldots,\right. \\
\left.\frac{\partial^{n+m-1} u}{\partial x^{n} \partial y^{m-1}}, \frac{\partial^{m} u}{\partial y^{m}}, \ldots, \frac{\partial^{n-1+m}}{\partial x^{n-1} \partial y^{m}}\right)
\end{aligned}
$$

$f \in C\left[I_{a b} \times R^{n m+n+m}, R\right], \alpha_{j} \in C^{(n)}\left[I_{a}, R\right], \beta_{i} \in C^{(m)}\left[I_{b}, R\right]$, and

$$
9 \alpha_{j}^{(i)}(0)=\beta_{i}^{(j)}(0) ; \quad 0 \leq j \leq m-1,0 \leq i \leq n-1 .
$$

It is well recognised that the method of upper and lower solutions, together with uniformly monotone convergent technique offers effective tools in proving and constructing multiple solutions of nonlinear problems. The upper and lower solutions that generate an interval in a suitable partially ordered space serve as upper and lower bounds for solutions which can be improved by uniformly monotone convergent iterative procedures. Obviously, from the computational point of view, monotone convergence has superiority over ordinary convergence and several monotonic iterative schemes for ordinary and partial differential equations have been developed and analysed in the monograph [9] (it covers more than 100 recent publications on the subject), and the papers $[5-7,10,11]$. In this paper we shall extend this fruitful technology for the initial value problem (1.1)-(1.4). Existence and uniqueness results for the partial differential equation (1.1) together with (1.2)-(1.3) or with different conditions have been considered by Agarwal [4], Lucia [12], Kovac [8] and Volpato [14], whereas linear and nonlinear Gronwall's and Wendroff's type inequalities, which are directly useful in estimating upper estimates of the solutions of $(1.1)-(1.4)$, are available in $[1,2]$.

\section{Hyperbolic differential inequalities}

We begin with the following

Definition 2.1. A function $w \in C^{(n, m)}\left[I_{a b}, R\right]$ is said to be an upper solution of the initial value problem (1.1)-(1.4) if

$$
\begin{array}{ll}
\frac{\partial^{n+m} w}{\partial x^{n} \partial y^{m}} \geq f(x, y,\langle w\rangle), & (x, y) \in I_{a b}, \\
\frac{\partial^{n+j} w(x, 0)}{\partial x^{n} \partial y^{j}} \geq \frac{\partial^{n+j} u(x, 0)}{\partial x^{n} \partial y^{j}}, & x \in I_{a}, \\
\frac{\partial^{i+m} w(0, y)}{\partial x^{i} \partial y^{m}} \geq \frac{\partial^{i+m} u(0, y)}{\partial x^{i} \partial y^{m}}, & y \in I_{b}, \\
\frac{\partial^{i+j} w(0,0)}{\partial x^{i} \partial y^{j}} \geq \frac{\partial^{i+j} u(0,0)}{\partial x^{i} \partial y^{j}}, & 0 \leq i \leq n-1,0 \leq j \leq m-1 .
\end{array}
$$


Conditions (2.2)-(2.4) are equivalent to the following conditions:

$$
\begin{array}{ll}
\frac{\partial^{i+j} w(x, 0)}{\partial x^{i} \partial y^{j}} \geq \frac{\partial^{i+j} u(x, 0)}{\partial x^{i} \partial y^{j}}, & 0 \leq i \leq n, 0 \leq j \leq m-1, \\
\frac{\partial^{i+j} w(0, y)}{\partial x^{i} \partial y^{j}} \geq \frac{\partial^{i+j} u(0, y)}{\partial x^{i} \partial y^{j}}, & 0 \leq i \leq n-1,0 \leq j \leq n .
\end{array}
$$

Similarly, a function $v \in C^{(n, m)}\left[I_{a b}, R\right]$ is said to be a lower solution of (1.1)(1.4) if for the function $v$ the reversed inequalities hold in (2.1)-(2.4).

LEMMA 2.1. If

$$
\frac{\partial^{n+m-1} w}{\partial x^{n} \partial y^{m-1}} \geq \frac{\partial^{n+m-1} u}{\partial x^{n} \partial y^{m-1}}, \quad(x, y) \in I_{a b},
$$

then conditions (2.2)-(2.4), or equivalently (2.5), (2.6) imply that

$$
\frac{\partial^{\mu+\nu} w}{\partial x^{\mu} \partial y^{\nu}} \geq \frac{\partial^{\mu+\nu} u}{\partial x^{\mu} \partial y^{\nu}}, \quad(x, y) \in I_{a b}, \quad 0 \leq \mu \leq n, 0 \leq \nu \leq m-1 .
$$

Proof. There exists a function $\lambda(x, y) \geq 0,(x, y) \in I_{a b}$ such that

$$
\frac{\partial^{n+m-1} \eta}{\partial x^{n} \partial y^{m-1}}=\lambda(x, y)
$$

where $\eta(x, y)=w(x, y)-u(x, y)$.

From (2.7) we easily find that

$$
\begin{aligned}
\frac{\partial^{\mu+\nu} \eta}{\partial x^{\mu} \partial y^{\nu}}= & \sum_{i=\mu}^{n-1} \frac{x^{i-\mu}}{(i-\mu) !} \frac{\partial^{i+\nu} \eta(0, y)}{\partial x^{i} \partial y^{\nu}} \\
& +\frac{1}{(n-\mu-1) !} \int_{0}^{x}(x-s)^{n-\mu-1}\left(\sum_{j=\nu}^{m-2} \frac{y^{j-\nu}}{(j-\nu) !} \frac{\partial^{j+j} \eta(s, 0)}{\partial s^{n} \partial y^{j}}\right) d s \\
& +\frac{1}{(n-\mu-1) !(m-1-\nu-1) !} \\
& \times \int_{0}^{x} \int_{0}^{y}(x-s)^{n-\mu-1}(y-t)^{m-1-\nu-1} \lambda(s, t) d t d s
\end{aligned}
$$

Since the right-hand side of $(2.8)$ is positive, the conclusion follows.

LEMMA 2.2. If $\partial^{n-1+m} w / \partial x^{n-1} \partial y^{m} \geq \partial^{n-1+m} u / \partial x^{n-1} \partial y^{m},(x, y) \in I_{a b}$, then conditions (2.2)-(2.4), or equivalently (2.5), (2.6) imply that

$$
\frac{\partial^{\mu+\nu} w}{\partial x^{\mu} \partial y^{\nu}} \geq \frac{\partial^{\mu+\nu} u}{\partial x^{\mu} \partial y^{\nu}} ; \quad(x, y) \in I_{a b}, \quad 0 \leq \mu \leq n-1,0 \leq \nu \leq m .
$$


The following fundamental result on hyperbolic differential inequalities includes several known results for the particular case $n=m=1$ obtained in $[7-11,13,15]$.

\section{THEOREM 2.3. Suppose that}

(i) $v, w \in C^{(n, m)}\left[I_{a b}, R\right]$, and

$$
\begin{gathered}
\frac{\partial^{n+m} v}{\partial x^{n} \partial y^{m}} \leq f(x, y,\langle v\rangle), \quad \frac{\partial^{n+m} w}{\partial x^{n} \partial y^{m}} \geq f(x, y,\langle w\rangle), \\
\frac{\partial^{n+j} v(x, 0)}{\partial x^{n} \partial y^{j}} \leq \frac{\partial^{n+j} w(x, 0)}{\partial x^{n} \partial y^{j}}, \\
\frac{\partial^{i+m} v(0, y)}{\partial x^{i} \partial y^{m}} \leq \frac{\partial^{i+m} w(0, y)}{\partial x^{i} \partial y^{m}}, \\
\frac{\partial^{i+j} v(0,0)}{\partial x^{i} \partial y^{j}} \leq \frac{\partial^{i+j} w(0,0)}{\partial x^{i} \partial y^{j}} ; \quad 0 \leq i \leq n-1,0 \leq j \leq m-1 ;
\end{gathered}
$$

(ii) $f\left(x, y, u_{0,0}, u_{1,0}, \ldots, u_{n-1, m}\right)$ is nondecreasing in $\left(u_{0,0}, u_{1,0}, \ldots, u_{u-1, m}\right)$;

(iii) $f\left(x, y, u_{0,0}, u_{1,0}, \ldots, u_{n-1, m}\right)-f\left(x, y, \bar{u}_{0,0}, \bar{u}_{1,0}, \ldots, \bar{u}_{n-1, m}\right) \leq$ $\sum_{i=0}^{n} \sum_{j=0}^{\prime m} L_{i, j}\left(u_{i, j}-\tilde{u}_{i, j}\right)$, whenever $u_{i, j} \geq \bar{u}_{i, j}$ on $I_{a b}$, where' deletes the choice $i=n$ and $j=m$, and $L_{i, j}$ are nonnegative constants. Then, the following hold

$$
\frac{\partial^{\mu+\nu} v}{\partial x^{\mu} \partial y^{\nu}} \leq \frac{\partial^{\mu+\nu} w}{\partial x^{\mu} \partial y^{\nu}} ; \quad 0 \leq \mu \leq n, 0 \leq \nu \leq m, \mu+\nu<n+m \text { on } I_{a b}
$$

Proof. We begin with the strict inequalities in (i) and prove the conclusion in (2.9) for strict inequalities. From Lemmas 2.1 and 2.2 it suffices to prove that

(2.10) $\frac{\partial^{n+m-1} v}{\partial x^{n} \partial y^{m-1}}<\frac{\partial^{n+m-1} w}{\partial x^{n} \partial y^{m-1}}$ and $\frac{\partial^{n-1+m} v}{\partial x^{n-1} \partial y^{m}}<\frac{\partial^{n-1+m} w}{\partial x^{n-1} \partial y^{m}}$ on $I_{a b}$.

Suppose that $(2.10)$ is false. Let $r_{0}$ be the greatest lower bound of numbers $r>x+y$ such that $(2.10)$ holds for $x+y<r_{0}$. Then, there is a point $\left(x_{0}, y_{0}\right) \in I_{a b}$ with $x_{0}+y_{0}=r_{0}$, and (say)

$$
\frac{\partial^{n+m-1} v\left(x_{0}, y_{0}\right)}{\partial x^{n} \partial y^{m-1}}=\frac{\partial^{n+m-1} w\left(x_{0}, y_{0}\right)}{\partial x^{n} \partial y^{m-1}} \text {. }
$$

Clearly, by hypothesis (i) (with strict inequalities), $y_{0}>0$. Also, for all $y \in\left[0, y_{0}\right]$

$$
\frac{\partial^{\mu+\nu} v\left(x_{0}, y\right)}{\partial x^{\mu} \partial y^{\nu}} \leq \frac{\partial^{\mu+\nu} w\left(x_{0}, y\right)}{\partial x^{\mu} \partial y^{\nu}} ; \quad 0 \leq \mu \leq n, 0 \leq \nu \leq m, \mu+\nu<n+m .
$$


Therefore, hypotheses (i) and (ii) yield the following contradiction to (2.11)

$$
\begin{aligned}
\frac{\partial^{n+m-1} v\left(x_{0}, y_{0}\right)}{\partial x^{n} \partial y^{m-1}} & \leq \frac{\partial^{n+m-1} v\left(x_{0}, 0\right)}{\partial x^{n} \partial y^{m-1}}+\int_{0}^{y_{0}} f\left(x_{0}, t,\left\langle v\left(x_{0}, t\right)\right\rangle\right) d t \\
& \leq \frac{\partial^{n+m-1} w\left(x_{0}, 0\right)}{\partial x^{n} \partial y^{m-1}}+\int_{0}^{y_{0}} f\left(x_{0}, t,\left\langle w\left(x_{0}, t\right)\right\rangle\right) d t \\
& \leq \frac{\partial^{n+m-1} w\left(x_{0}, y_{0}\right)}{\partial x^{n} \partial y^{m-1}}
\end{aligned}
$$

A similar contradiction can be arrived at if we assume

$$
\frac{\partial^{n-1+m} v\left(x_{0}, y_{0}\right)}{\partial x^{n-1} \partial y^{m}}=\frac{\partial^{n-1+m} w\left(x_{0}, y_{0}\right)}{\partial x^{n-1} \partial y^{m}} \text {. }
$$

Therefore (2.10) and consequently (2.9) with strict inequalities hold.

Now, let $\varepsilon>0$. Choose $\lambda>0$ such that

$$
\lambda^{n+m}-\sum_{i=0}^{n} \sum_{j=0}^{m} L_{i, j} \lambda^{i+j}>0 .
$$

A $\lambda>0$ satisfying (2.12) always exists.

We consider the functions

$$
v(x, y)=v(x, y)-\varepsilon e^{\lambda(x+y)} \quad \text { and } \quad \bar{w}(x, y)=w(x, y)+\varepsilon e^{\lambda(x+y)} .
$$

Then, from hypotheses (ii), (iii) and the choice of $\lambda$ in (2.12), we obtain

$$
\begin{aligned}
\frac{\partial^{n+m} \bar{w}}{\partial x^{n} \partial y^{m}} & \geq f(x, y,\langle w\rangle)+\lambda^{n+m} \varepsilon e^{\lambda(x+y)} \\
& \geq f(x, y,\langle\bar{w}\rangle)+\varepsilon e^{\lambda(x+y)}\left[\lambda^{n+m}-\sum_{i=0}^{n} \sum_{j=0}^{m} L_{i, j} \lambda^{i+j}\right] \\
& >f(x, y,\langle\bar{w}\rangle) .
\end{aligned}
$$

Similarly, it can be shown that

$$
\frac{\partial^{n+m} v}{\partial x^{n} \partial y^{m}}<f(x, y,\langle\bar{v}\rangle)
$$

Further, it is obvious that

$$
\begin{aligned}
\frac{\partial^{n+j} v(x, 0)}{\partial x^{n} \partial y^{j}}<\frac{\partial^{n+j} \bar{w}(x, 0)}{\partial x^{n} \partial y^{j}}, & \frac{\partial^{i+m} \bar{v}(0, y)}{\partial x^{i} \partial y^{m}}<\frac{\partial^{i+m} \bar{w}(0, y)}{\partial x^{i} \partial y^{m}} \\
\frac{\partial^{i+j} v(0,0)}{\partial x^{i} \partial y^{j}}<\frac{\partial^{i+j} \bar{w}(0,0)}{\partial x^{i} \partial y^{j}} ; & 0 \leq i \leq n-1,0 \leq j \leq m-1 .
\end{aligned}
$$

Thus, from the first part it follows that

$$
\frac{\partial^{\mu+\nu} v}{\partial x^{\mu} \partial y^{\nu}}<\frac{\partial^{\mu+\nu} w}{\partial x^{\mu} \partial y^{\nu}} ; \quad 0 \leq \mu \leq n, 0 \leq \nu \leq m, \mu+\nu<n+m \text { on } I_{a b}
$$


The conclusion (2.9) is obtained by letting $\varepsilon \rightarrow 0$ in (2.14).

In our next result we shall dispense with the assumption of monotonicity of $f$ at the cost of strengthening the notion of lower and upper solutions.

THEOREM 2.4. Suppose that

(i) $v, w \in C^{(n, m)}\left[I_{a b}, R\right]$ are such that $\partial^{\mu+\nu} v / \partial x^{\mu} \partial y^{\nu} \leq \partial^{\mu+\nu} w / \partial x^{\mu} \partial y^{\nu}$; $0 \leq \mu \leq n, 0 \leq \nu \leq m, \mu+\nu<n+m$ on $I_{a b}$;

(ii)

$$
\begin{aligned}
\frac{\partial^{n+m} v}{\partial x^{n} \partial y^{m}} & \leq f\left(x, y, u_{0,0}, u_{1,0}, \ldots, u_{n-1, m}\right) \\
\frac{\partial^{n+m} w}{\partial x^{n} \partial y^{m}} & \geq f\left(x, y, u_{0,0}, u_{1,0}, \ldots, u_{n-1, m}\right)
\end{aligned}
$$

whenever

$$
\frac{\partial^{\mu+\nu} v}{\partial x^{\mu} \partial y^{\nu}} \leq u_{\mu, \nu} \leq \frac{\partial^{\mu+\nu} w}{\partial x^{\mu} \partial y^{\nu}} \quad \text { on } I_{a b}
$$

(iii)

$$
\begin{aligned}
\frac{\partial^{n+j} v(x, 0)}{\partial x^{n} \partial x^{j}} \leq \alpha_{j}^{(n)}(x) & \leq \frac{\partial^{n+j} w(x, 0)}{\partial x^{n} \partial y^{j}}, \\
\frac{\partial^{i+m} v(0, y)}{\partial x^{i} \partial y^{m}} \leq \beta_{i}^{(m)}(y) & \leq \frac{\partial^{i+m} w(0, y)}{\partial x^{i} \partial y^{m}}, \\
\frac{\partial^{i+j} v(0,0)}{\partial x^{i} \partial y^{j}} \leq \alpha_{i}^{(j)}(0) & \leq \frac{\partial^{i+j} w(0,0)}{\partial x^{i} \partial x^{j}} ; \\
& 0 \leq i \leq n-1,0 \leq j \leq m-1 ;
\end{aligned}
$$

(iv) $f$ satisfies the Lipschitz condition on $I_{a b} \times R^{n m+n+m}$

$$
\begin{aligned}
& \left|f\left(x, y, u_{0,0}, u_{1,0}, \ldots, u_{n-1, m}\right)-f\left(x, y, \bar{u}_{0,0}, \bar{u}_{1,0}, \ldots, \bar{u}_{n-1, m}\right)\right| \\
& \quad \leq \sum_{i=0}^{n} \sum_{j=0}^{m} L_{i, j}\left|u_{i, j}-\bar{u}_{i, j}\right| .
\end{aligned}
$$

Then, for any solution (only solution [4]) $u$ of the initial value problem (1.1)(1.3) the following hold

$$
\begin{aligned}
\frac{\partial^{\mu+\nu} v}{\partial x^{\mu} \partial y^{\nu}} \leq \frac{\partial^{\mu+\nu} u}{\partial x^{\mu} \partial y^{\nu}} & \leq \frac{\partial^{\mu+\nu} w}{\partial x^{\mu} \partial y^{\nu}} \\
0 & \leq \mu \leq n, 0 \leq \nu \leq m, \mu+\nu<n+m \text { on } I_{a b} .
\end{aligned}
$$

Proof. As in Theorem 2.3, if $v$ and $w$ satisfy strict inequalities in the hypotheses (ii) and (iii) then strict inequalities follow in the conclusion (2.17). 
Now, let $\varepsilon>0$ and $\lambda>0$ be such that the inequality (2.12) holds, and consider the functions $v$ and $\bar{w}$ defined in (2.13). We also define the functions $A_{\mu, \nu}(x, y, u): I_{a b} \times R \rightarrow R ; 0 \leq \mu \leq n, 0 \leq \nu \leq m, \mu+\nu<n+m$, as follows

$$
A_{\mu, \nu}(x, y, u)=\max \left\{\frac{\partial^{\mu+\nu} v}{\partial x^{\mu} \partial y^{\nu}}, \min \left\{u, \frac{\partial^{\mu+\nu} w}{\partial x^{\mu} \partial y^{\nu}}\right\}\right\} .
$$

Let $\bar{z}=\bar{z}(x, y)$ be such that

$$
\begin{aligned}
\frac{\partial^{\mu+\nu} v}{\partial x^{\mu} \partial y^{\nu}} \leq \frac{\partial^{\mu+\nu} \bar{z}}{\partial x^{\mu} \partial y^{\nu}} \leq \frac{\partial^{\mu+\nu} \bar{w}}{\partial x^{\mu} \partial y^{\nu}} & \\
0 & \leq \mu \leq n, 0 \leq \nu \leq m, \mu+\nu<n+m \text { on } I_{a b} .
\end{aligned}
$$

Then, $u_{\mu+\nu}=A_{\mu, \nu}\left(x, y, \partial^{\mu+\nu} \bar{z} / \partial x^{\mu} \partial y^{\nu}\right) ; 0 \leq \mu \leq n, 0 \leq \nu \leq m, \mu+\nu<$ $n+m$, satisfy

$$
\begin{aligned}
\frac{\partial^{\mu+\nu} v}{\partial x^{\mu} \partial y^{\nu}} \leq u_{\mu+\nu} & \leq \frac{\partial^{\mu+\nu} w}{\partial x^{\mu} \partial y^{\nu}} \\
& 0 \leq \mu \leq n, 0 \leq \nu \leq m, \mu+\nu<n+m \text { on } I_{a b},
\end{aligned}
$$

and also,

$$
\left|u_{\mu+\nu}-\frac{\partial^{\mu+\nu} \bar{z}}{\partial x^{\mu} \partial y^{\nu}}\right| \leq \varepsilon \lambda^{\mu+\nu} e^{\lambda(x+y)} .
$$

Consequently, from the hypotheses (ii) and (iv), we find

$$
\begin{aligned}
\frac{\partial^{n+m} w}{\partial x^{n} \partial y^{m}} & \geq f\left(x, y, u_{0,0}, u_{1,0}, \ldots, u_{n-1, m}\right)+\lambda^{n+m} \varepsilon e^{\lambda(x+y)} \\
& \geq-\sum_{i=0}^{n} \sum_{j=0}^{m} L_{i, j}\left|u_{i, j}-\frac{\partial^{i+j} \bar{z}}{\partial x^{i} \partial y^{j}}\right|+\lambda^{n+m} \varepsilon e^{\lambda(x+y)}+f(x, y,\langle\bar{z}\rangle) \\
& \geq \varepsilon e^{\lambda(x+y)}\left[\lambda^{n+m}-\sum_{i=0}^{n} \sum_{j=0}^{m} L_{i, j} \lambda^{i+j}\right]+f(x, y,\langle\bar{z}\rangle) \\
& >f(x, y,\langle\bar{z}\rangle)
\end{aligned}
$$

for all $\langle\bar{z}\rangle$ satisfying (2.19). Similarly, the inequality $\partial^{n+m} \bar{v} / \partial x^{n} \partial y^{m}<$ $f(x, y,\langle\bar{v}\rangle)$ can be proved. Further, since

$$
\begin{aligned}
& \frac{\partial^{n+j} v(x, 0)}{\partial x^{n} \partial y^{j}}<\alpha_{j}^{(n)}(x)<\frac{\partial^{n+j} \bar{w}(x, 0)}{\partial x^{n} \partial y^{j}}, \\
& \frac{\partial^{i+m} v(0, y)}{\partial x^{i} \partial y^{m}}<\beta_{i}^{(m)}(y)<\frac{\partial^{i+m} \bar{w}(0, y)}{\partial x^{i} \partial y^{m}}, \\
& \frac{\partial^{i+j} v(0,0)}{\partial x^{i} \partial y^{j}}<\alpha_{i}^{(j)}(0)<\frac{\partial^{i+j} \bar{w}(0,0)}{\partial x^{i} \partial y^{j}} ; \quad 0 \leq i \leq n-1,0 \leq j \leq m-1 ;
\end{aligned}
$$


from the first part it follows that

$$
\begin{aligned}
\frac{\partial^{\mu+\nu} v}{\partial x^{\mu} \partial y^{\nu}}< & \frac{\partial^{\mu+\nu} u}{\partial x^{\mu} \partial y^{\nu}}<\frac{\partial^{\mu+\nu} w}{\partial x^{\mu} \partial y^{\nu}} \\
& 0 \leq \mu \leq n, 0 \leq \nu \leq m, \mu+\nu<n+m \text { on } I_{a b} .
\end{aligned}
$$

The conclusion (2.17) follows by letting $\varepsilon \rightarrow 0$ in (2.22).

COROLlary 2.5. Suppose that

(i) $v, w \in C^{(n, m)}\left[I_{a b}, R\right]$ are, respectively, the lower and upper solutions of the initial value problem (1.1)-(1.3);

(ii) hypothesis (ii) of Theorem 2.3 holds;

(iii) hypothesis (iv) of Theorem 2.4 holds.

Then, the conclusion (2.17) holds.

\section{Existence results}

THEOREM 3.1. Suppose that

(i) $v, w \in C^{(n, m)}\left[I_{a b}, R\right]$ are, respectively, the lower and upper solutions of the initial value problem (1.1)-(1.3), satisfying

$$
\frac{\partial^{\mu+\nu} v}{\partial x^{\mu} \partial y^{\nu}} \leq \frac{\partial^{\mu+\nu} w}{\partial x^{\mu} \partial y^{\nu}} ; \quad 0 \leq \mu \leq n, 0 \leq \nu \leq m, \mu+\nu<n+m \text { on } I_{a b}
$$

(ii) hypothesis (ii) of Theorem 2.3 holds.

Then, there exists a solution $u(x, y)$ of the initial value problem (1.1)-(1.3), satisfying (2.17).

Proof. Let $A_{\mu, \nu}(x, y, u) ; 0 \leq \mu \leq n, 0 \leq \nu \leq m, \mu+\nu<n+m$, be the functions as defined in (2.18). Then, the function $f\left(x, y, A_{0,0}(x, y, u)\right.$, $\left.A_{1,0}(x, y, u), \ldots, A_{n-1, m}(x, y, u)\right)$ defines a continuous extension of $f$ to $I_{a b} \times$ $R^{n m+n+m}$, and it is bounded. Therefore, the modified differential equation

$$
\frac{\partial^{n+m} u}{\partial x^{n} \partial y^{m}}=f\left(x, y, A_{0,0}(x, y, u), A_{1,0}(x, y, u), \ldots, A_{n-1, m}(x, y, u)\right)
$$

together with the initial conditions (1.2), (1.3) has a solution $\bar{u}(x, y)$ on $I_{a b}$ [4]. It therefore suffices to show that

$$
\begin{aligned}
\frac{\partial^{\mu+\nu} v}{\partial x^{\mu} \partial y^{\nu}} & \leq \frac{\partial^{\mu+\nu} \bar{u}}{\partial x^{\mu} \partial y^{\nu}} \leq \frac{\partial^{\mu+\nu} w}{\partial x^{\mu} \partial y^{\nu}} \\
0 & \leq \mu \leq n, 0 \leq \nu \leq m, \mu+\nu<n+m \text { on } I_{a b} .
\end{aligned}
$$

For $\varepsilon>0$, we define the functions

$$
\bar{v}(x, y)=v(x, y)-\varepsilon(1+x)^{n}(1+y)^{m}, \quad \bar{w}(x, y)=w(x, y)+\varepsilon(1+x)^{n}(1+y)^{m} .
$$


We claim that

$$
\begin{aligned}
\frac{\partial^{\mu+\nu} v}{\partial x^{\mu} \partial y^{\nu}}<\frac{\partial^{\mu+\nu} \bar{a}}{\partial x^{\mu} \partial y^{\nu}}<\frac{\partial^{\mu+\nu} \bar{w}}{\partial x^{\mu} \partial y^{\nu}} & \\
0 & \leq \mu \leq n, 0 \leq \nu \leq m, \mu+\nu<n+m \text { on } I_{a b} .
\end{aligned}
$$

Since

$$
\begin{aligned}
& \frac{\partial^{n+j} v(x, 0)}{\partial x^{n} \partial y^{j}}<\frac{\partial^{n+j} \bar{u}(x, 0)}{\partial x^{n} \partial y^{j}}<\frac{\partial^{n+j} \bar{w}(x, 0)}{\partial x^{n} \partial y^{j}}, x \in I_{a}, \\
& \frac{\partial^{i+m} v(0, y)}{\partial x^{i} \partial y^{m}}<\frac{\partial^{i+m} \bar{u}(0, y)}{\partial x^{i} \partial y^{m}}<\frac{\partial^{i+m} \bar{w}(0, y)}{\partial x^{i} \partial y^{m}}, \quad y \in I_{b}, \\
& \frac{\partial^{i+j} v(0,0)}{\partial x^{i} \partial y^{j}}<\frac{\partial^{i+j} \bar{u}(0,0)}{\partial x^{i} \partial y^{j}}<\frac{\partial^{i+j} \bar{w}(0,0)}{\partial x^{i} \partial y^{j}}, \\
& 0 \leq i \leq n-1,0 \leq j \leq m-1,
\end{aligned}
$$

from Lemmas 2.1 and 2.2 it suffices to show that

$$
\frac{\partial^{n+m-1} \bar{v}}{\partial x^{n} \partial y^{m-1}}<\frac{\partial^{n+m-1} \bar{u}}{\partial x^{n} \partial y^{m-1}}<\frac{\partial^{n+m-1} \bar{w}}{\partial x^{n} \partial y^{m-1}}
$$

and

$$
\frac{\partial^{n-1+m} v}{\partial x^{n-1} \partial y^{m}}<\frac{\partial^{n-1+m} \bar{u}}{\partial x^{n-1} \partial y^{m}}<\frac{\partial^{n-1+m} \bar{w}}{\partial x^{n-1} \partial y^{m}} \text { on } I_{a b}
$$

Let $r_{0}$ be the greatest lower bound of numbers $r>x+y$ such that (3.5) and (3.6) holds for $x+y<r_{0}$. Then, there is a point $\left(x_{0}, y_{0}\right) \in I_{a b}$ with $x_{0}+y_{0}=r_{0}$, and (say)

$$
\frac{\partial^{n+m-1} \tilde{v}\left(x_{0}, y_{0}\right)}{\partial x^{n} \partial y^{m-1}}=\frac{\partial^{n+m-1} \bar{u}\left(x_{0}, y_{0}\right)}{\partial x^{n} \partial y^{m-1}} .
$$

Clearly, by (3.4), $y_{0}>0$. Also, for all $y \in\left[0, y_{0}\right]$ we have

$$
\begin{aligned}
\frac{\partial^{\mu+\nu} \bar{v}\left(x_{0}, y\right)}{\partial x^{\mu} \partial y^{\nu}} \leq \frac{\partial^{\mu+\nu} \bar{u}\left(x_{0}, y\right)}{\partial x^{\mu} \partial y^{\nu}} \leq & \frac{\partial^{\mu+\nu} \bar{w}\left(x_{0}, y\right)}{\partial x^{\mu} \partial y^{\nu}} ; \\
& 0 \leq \mu \leq n, 0 \leq \nu \leq m, \mu+\nu<n+m .
\end{aligned}
$$

Further, from the definition of $A_{\mu, \nu}(x, y, u)$ in $(2.18)$, for all $y \in\left[0, y_{0}\right]$ we also have

$$
\begin{aligned}
\frac{\partial^{\mu+\nu} v\left(x_{0}, y\right)}{\partial x^{\mu} \partial y^{\nu}} \leq A_{\mu, \nu}\left(x_{0}, y, \frac{\partial^{\mu+\nu} \bar{u}\left(x_{0}, y\right)}{\partial x^{\mu} \partial y^{\nu}}\right) & \leq \frac{\partial^{\mu+\nu} w\left(x_{0}, y\right)}{\partial x^{\mu} \partial y^{\nu}} \\
0 & \leq \mu \leq m, 0 \leq \nu \leq m, \mu+\nu<n+m .
\end{aligned}
$$

Therefore, hypothesis (ii) yields the following contradiction to (3.7): 


$$
\begin{aligned}
& \frac{\partial^{n+m-1} \bar{u}\left(x_{0}, y_{0}\right)}{\partial x^{n} \partial y^{m-1}}=\frac{\partial^{n+m-1} \bar{u}\left(x_{0}, 0\right)}{\partial x^{n} \partial y^{m-1}} \\
& \quad+\int_{0}^{y_{0}} f\left(x_{0}, t, A_{0,0}\left(x_{0}, t, \bar{u}\left(x_{0}, t\right)\right), A_{1,0}\left(x_{0}, t, \frac{\partial \bar{u}\left(x_{0}, t\right)}{\partial x}\right)\right. \\
& \left.\quad \ldots, A_{n-1, m}\left(x_{0}, t, \frac{\partial^{n-1, m} \bar{u}\left(x_{0}, t\right)}{\partial x^{n-1} \partial t^{m}}\right)\right) d t \\
& \geq \frac{\partial^{n+m-1} v\left(x_{0}, 0\right)}{\partial x^{n} \partial y^{m-1}}+\int_{0}^{y_{0}} f\left(x_{0}, t,\left\langle v\left(x_{0}, t\right)\right\rangle\right) d t \\
& \geq \frac{\partial^{n+m-1} v\left(x_{0}, y_{0}\right)}{\partial x^{n} \partial y^{m-1}}>\frac{\partial^{n+m-1} \bar{v}\left(x_{0}, y_{0}\right)}{\partial x^{n} \partial y^{m-1}} .
\end{aligned}
$$

A similar contradiction holds if instead of (3.7) we assume any one of the other three possibilities in (3.5) and (3.6).

Thus, the inequalities (3.5) and (3.6) hold. The conclusion now follows by letting $\varepsilon \rightarrow 0$ in (3.3).

COROLlary 3.2. Suppose that the hypotheses (i)-(iii) of Theorem 2.4 are satisfied. Then, there exists a solution $u(x, y)$ of the initial value problem (1.1)-(1.3), satisfying (2.17).

\section{Variation of parameters formula}

THEOREM 4.1. Any solution (only solution) $u(x, y)$ on $I_{a b}$ of the linear differential equation

$$
\frac{\partial^{n+m} u}{\partial x^{n} \partial y^{m}}=-\sum_{i=0}^{n} \sum_{j=0}^{m}\left(\begin{array}{c}
n \\
i
\end{array}\right)\left(\begin{array}{c}
m \\
j
\end{array}\right)\left(-M_{1}\right)^{n-i}\left(-M_{2}\right)^{m-j} \frac{\partial^{i+j} u}{\partial x^{i} \partial y^{j}}+h(x, y)
$$

together with (1.2)-(1.4) can be written as

$$
u(x, y)=\varphi(x, y)+\psi(x, y)
$$


where $M_{1}$ and $M_{2}$ are constants, and the functions $\varphi(x, y), \psi(x, y)$ are defined as follows

(4.3)

$$
\begin{aligned}
& \varphi(x, y)=e^{M_{1} x} \sum_{i=0}^{n-1} \frac{x^{i}}{i !}\left(\sum_{k=0}^{n-i-1} \frac{\left(-x M_{1}\right)^{k}}{k !}\right) \beta_{i}(y) \\
& +e^{M_{2} y} \sum_{j=0}^{m-1} \frac{y^{j}}{j !}\left(\sum_{l=0}^{n-i-1} \frac{\left(-y M_{2}\right)^{l}}{l !}\right) \alpha_{j}(x) \\
& -e^{M_{1} x+M_{2} y} \sum_{i=0}^{n-1} \sum_{j=0}^{m-1} \frac{x^{i}}{i !} \frac{y^{j}}{j !}\left(\sum_{k=0}^{n-i-1} \frac{\left(-x M_{1}\right)^{k}}{k !}\right)\left(\sum_{l=0}^{n-j-1} \frac{\left(-y M_{2}\right)^{l}}{l !}\right) \alpha_{j}^{(i)}(0), \\
& \psi(x, y)=e^{M_{1} x+M_{2} y} \frac{1}{(n-1) !(m-1) !} \\
& \times \int_{0}^{x} \int_{0}^{y}(x-s)^{n-1}(y-t)^{m-1} e^{-M_{1} s-M_{2} t} h(s, t) d t d s .
\end{aligned}
$$

Proof. Let us assume that any solution of the linear differential equation (4.1) can be written as

$$
u(x, y)=e^{M_{1} x+M_{2} y} z(x, y)
$$

from which it is easy to obtain that

$$
\frac{\partial^{n+m} z}{\partial x^{n} \partial y^{m}}=e^{-M_{1} x-M_{2} y} \sum_{i=0}^{n} \sum_{j=0}^{m}\left(\begin{array}{c}
n \\
i
\end{array}\right)\left(\begin{array}{c}
m \\
j
\end{array}\right)\left(-M_{1}\right)^{n-i}\left(-M_{2}\right)^{m-j} \frac{\partial^{i+j} u}{\partial x^{i} \partial y^{j}}
$$

and hence

$$
\begin{aligned}
\frac{\partial^{n+m} u}{\partial x^{n} \partial y^{m}}= & -\sum_{i=0}^{n} \sum_{j=0}^{m}\left(\begin{array}{c}
n \\
i
\end{array}\right)\left(\begin{array}{c}
m \\
j
\end{array}\right)\left(-M_{1}\right)^{n-i}\left(-M_{2}\right)^{m-j} \frac{\partial^{i+j} u}{\partial x^{i} \partial y^{j}} \\
& +e^{M_{1} x+M_{2} y} \frac{\partial^{n+m} z}{\partial x^{n} \partial y^{m}} .
\end{aligned}
$$

Thus, $u(x, y)$ defined in (4.5) is a solution of (4.1) if and only if

$$
e^{M_{1} x+M_{2} y} \frac{\partial^{n+m} z}{\partial x^{n} \partial y^{m}}=h(x, y) .
$$

From (4.6) it is easy to find that

$$
\begin{aligned}
z(x, y) & =\sum_{i=0}^{n-1} \frac{x^{i}}{i !} \frac{\partial^{i} z(0, y)}{\partial x^{i}}+\frac{1}{(n-1) !} \int_{0}^{x}(x-s)^{n-1}\left(\sum_{j=0}^{m-1} \frac{y^{j}}{j !} \frac{\partial^{n+j} z(s, 0)}{\partial s^{n} \partial y^{j}}\right) d s \\
+ & \frac{1}{(n-1) !(m-1) !} \times \int_{0}^{x} \int_{0}^{y}(x-s)^{n-1}(y-t)^{m-1} e^{-M_{1} s-M_{2} t} h(s, t) d t d s .
\end{aligned}
$$


Hence, the solution $u(x, y)$ of the initial value problem (4.1), (1.2)-(1.4) can be written as

$$
\begin{aligned}
& u(x, y)=e^{M_{1} x+M_{2} y}\left[\sum_{i=0}^{n-1} \frac{x^{i}}{i !} \sum_{k=0}^{i}\left(\begin{array}{l}
i \\
k
\end{array}\right)\left(-M_{1}\right)^{i-k} e^{-M_{2} y} \beta_{k}(y)\right. \\
& +\frac{1}{(n-1) !} \int_{0}^{x}(x-s)^{n-1} \frac{\partial^{n}}{\partial s^{n}} \\
& \left.\times\left(\sum_{j=0}^{m-1} \frac{y^{j}}{j !} \sum_{l=0}^{j}\left(\begin{array}{l}
j \\
l
\end{array}\right)\left(-M_{2}\right)^{j-l} e^{-M_{1} s} \alpha_{l}(s)\right) d s\right] \\
& +\psi(x, y) \\
& =e^{M_{1} x} \sum_{i=0}^{n-1} \frac{x^{i}}{i !}\left(\sum_{k=0}^{n-i-1} \frac{\left(-x M_{1}\right)^{k}}{k !}\right) \beta_{i}(y) \\
& +e^{M_{1} x+M_{2} y} \frac{1}{(n-1) !} \sum_{j=0}^{m-1} \frac{y^{j}}{j !}\left(\sum_{l=0}^{m-j-1} \frac{\left(-y M_{2}\right)^{l}}{l !}\right) \\
& \times \int_{0}^{x}(x-s)^{n-1} \frac{\partial^{n}}{\partial s^{n}}\left(e^{-M_{1} s} \alpha_{j}(s)\right) d s+\psi(x, y) \\
& =\varphi(x, y)+\psi(x, y) \text {. }
\end{aligned}
$$

RemarK 4.1. From the definition of $\psi(x, y)$ in (4.4) it follows that

$$
\begin{aligned}
\frac{\partial^{\mu+\nu} \psi}{\partial x^{\mu} \partial y^{\nu}}= & e^{M_{1} x+M_{2} y} \sum_{i=0}^{\mu} \sum_{j=0}^{\nu}\left(\begin{array}{c}
\mu \\
i
\end{array}\right)\left(\begin{array}{c}
\nu \\
j
\end{array}\right) M_{1}^{\mu-i} M_{2}^{\nu-j} \frac{1}{(n-i-1) !(m-j-1) !} \\
& \times \int_{0}^{x} \int_{0}^{y}(x-s)^{n-i-1}(y-t)^{m-j-1} e^{-M_{1} s-M_{2} t} h(s, t) d t d s .
\end{aligned}
$$

Thus, for all nonnegative constants $M_{1}$ and $M_{2}$, and nonnegative function $h(x, y)$ on $I_{a b}, \partial^{\mu+\nu} \psi / \partial x^{\mu} \partial y^{\nu} \geq 0 ; 0 \leq \mu \leq n, 0 \leq \nu \leq m, \mu+\nu<n+m$ on $I_{a b}$.

ReMARK 4.2. From the explicit representation of $\varphi(x, y)$ in (4.3) it is possible to find sufficient conditions on the functions $\alpha_{j}(x)$ and $\beta_{i}(y)$ and their derivatives so that $\partial^{\mu+\nu} \varphi / \partial x^{\mu} \partial y^{\nu} \geq 0,0 \leq \mu \leq n, 0 \leq \nu \leq m, \mu+\nu<$ $n+m$ on $I_{a b}$. For example, if $n=m=1$, then (4.3) reduces to

$$
\varphi(x, y)=e^{M_{1} x} \beta_{0}(y)+e^{M_{2} y} \alpha_{0}(x)-e^{M_{1} x+M_{2} y} \alpha_{0}(0)
$$

and hence it suffices to assume that $M_{1}$ and $M_{2}$ are nonnegative constants, $\beta_{0}^{\prime}(y) \geq 0$ on $I_{b}, \alpha_{0}^{\prime}(x) \geq 0$ on $I_{a}$ and $\alpha_{0}(0)=\beta_{0}(0)=0$. As another example, 
if $M_{1}=M_{2}=0$, then it is easy to note that

$$
\begin{aligned}
\varphi(x, y) & =\sum_{i=0}^{n-1} \frac{x^{i}}{i !} \beta_{i}(y)+\sum_{j=0}^{m-1} \frac{y^{j}}{j !} \alpha_{j}(x)-\sum_{i=0}^{n-1} \sum_{j=0}^{m-1} \frac{x^{i}}{i !} \frac{y^{j}}{j !} \alpha_{j}^{(i)}(0) \\
& =\sum_{i=0}^{n-1} \frac{x^{i}}{i !} \beta_{i}(y)+\frac{1}{(n-1) !} \sum_{j=0}^{m-1} \frac{y^{j}}{j !} \int_{0}^{x}(x-s)^{n-1} \alpha_{j}^{(n)}(s) d s \\
& =\sum_{j=0}^{m-1} \frac{y^{j}}{j !} \alpha_{j}(x)+\frac{1}{(m-1) !} \sum_{i=0}^{n-1} \frac{x^{i}}{i !} \int_{0}^{y}(y-t)^{m-1} \beta_{i}^{(m)}(t) d t
\end{aligned}
$$

and hence it suffices to assume that $\beta_{i}^{(\nu)}(y) \geq 0,0 \leq \nu \leq m$ on $I_{b}$ and $\alpha_{i}^{(n)}(x) \geq 0$ on $I_{a}$, or equivalently $\alpha_{j}^{(\mu)}(x) \geq 0,0 \leq \mu \leq n$ on $I_{a}$ and $\beta_{i}^{(m)}(y) \geq$ 0 on $I_{b}$.

From Theorem 4.1 and Remarks 4.1 and 4.2 the following result can be proved immediately.

THEOREM 4.2. Suppose that

(i) $u \in C^{(n, m)}\left[I_{a b}, R\right]$ is such that

$$
\frac{\partial^{n+m} \bar{u}}{\partial x^{n} \partial y^{m}} \geq-\sum_{i=0}^{n} \sum_{j=0}^{m}\left(\begin{array}{c}
n \\
i
\end{array}\right)\left(\begin{array}{c}
m \\
j
\end{array}\right)\left(-M_{1}\right)^{n-i}\left(-M_{2}\right)^{m-j} \frac{\partial^{i+j} \bar{u}}{\partial x^{i} \partial y^{j}}
$$

where $M_{1}$ and $M_{2}$ are nonnegative constants

(ii) $\partial^{\mu+\nu} \varphi_{a} / \partial x^{\mu} \partial y^{\nu} \geq 0 ; 0 \leq \mu \leq n, 0 \leq \nu \leq m, \mu+\nu<n+m$ on $I_{a b}$, where $\varphi_{a}(x, y)$ is same as $\varphi(x, y)$ replacing $\alpha_{j}(x)$ by $\partial^{j} \vec{u}(x, 0) / \partial x^{j}$ and $\beta_{i}(y)$ by $\partial^{i} \bar{u}(0, y) / \partial x^{i}$.

Then, the following hold

$$
\frac{\partial^{\mu+\nu} \bar{u}}{\partial x^{\mu} \partial y^{\nu}} \geq 0, \quad 0 \leq \mu \leq n, 0 \leq \nu \leq m, \mu+\nu<n+m \text { on } I_{a b} .
$$

\section{Monotone iterative method}

The purpose of this section is to provide sufficient conditions so that the iterative scheme

$$
\begin{aligned}
& \frac{\partial^{n+m} u_{k+1}}{\partial x^{n} \partial y^{m}}=-\sum_{i=0}^{n} \sum_{j=0}^{m}\left(\begin{array}{c}
n \\
i
\end{array}\right)\left(\begin{array}{c}
m \\
j
\end{array}\right)\left(-M_{1}\right)^{n-i}\left(-M_{2}\right)^{m-j} \frac{\partial^{i+j} u_{k+1}}{\partial x^{i} \partial y^{j}} \\
& \quad+f\left(x, y,\left(u_{k}\right\rangle\right)+\sum_{i=0}^{m} \sum_{j=0}^{m}\left(\begin{array}{c}
n \\
i
\end{array}\right)\left(\begin{array}{c}
m \\
j
\end{array}\right)\left(-M_{1}\right)^{n-i}\left(-M_{2}\right)^{m-j} \frac{\partial^{i+j} u_{k}}{\partial x^{i} \partial y^{j}}
\end{aligned}
$$




$$
\begin{aligned}
\frac{\partial^{j} u_{k+1}(x, 0)}{\partial y^{j}}=\alpha_{j}(x), & & 0 \leq j \leq m-1, \\
\frac{\partial^{i} u_{k+1}(0, y)}{\partial x^{i}}=\beta_{i}(y), & & 0 \leq i \leq n-1,
\end{aligned}
$$

with the given function $u_{0}(x, y)$, generates the sequences $\left\{\partial^{\mu+\nu} u_{k} / \partial x^{\mu} \partial y^{\nu}\right\}$, $0 \leq \mu \leq n, 0 \leq \nu \leq m, \mu+\nu<n+m$, which converge uniformly and monotonically to $\partial^{\mu+\nu} u / \partial x^{\mu} \partial y^{\nu}$ on $I_{a b}$, where $u(x, y)$ is a solution of the initial value problem (1.1)-(1.4). For this, we will need the following lemma.

LEMMA 5.1 [3]. Let $(E, \leq)$ be a partially ordered space and $v_{0} \leq w_{0}$ be two elements of $E$. Denote by $\left[v_{0}, w_{0}\right]$ the interval $\left\{u \in E: v_{0} \leq u \leq w_{0}\right\}$. Let $T:\left[v_{0}, w_{0}\right] \rightarrow E$ be an isotone operator $(T(v) \leq T(w)$, whenever $v \leq w)$ and let it possess the properties

(i) $v_{0} \leq T\left(v_{0}\right)$,

(ii) the (nondecreasing) sequence $\left\{T^{k}\left(v_{0}\right)\right\}$ where $T^{0}\left(v_{0}\right)=v_{0}, T^{k+1}\left(v_{0}\right)=$ $T\left[T^{k}\left(v_{0}\right)\right]$ for each $k=0,1, \ldots$ is well defined, that is, $T^{k}\left(v_{0}\right) \leq w_{0}$ for each $k$,

(iii) the sequence $\left\{T^{k}\left(v_{0}\right)\right\}$ has $\sup v \in E$, that is, $T^{k}\left(v_{0}\right) \uparrow v$,

(iv) $T^{k+1}\left(v_{0}\right) \uparrow T(v)$,

(i) $T\left(w_{0}\right) \leq w_{0}$,

(ii)' the (nonincreasing) sequence $\left\{T^{k}\left(w_{0}\right)\right\}$ is well defined, that is, $T^{k}\left(w_{0}\right)$ $\geq v_{0}$ for each $k$,

(iii) the sequence $\left\{T^{k}\left(w_{0}\right)\right\}$ has inf $w \in E$, that is, $T^{k}\left(y_{0}\right) \downarrow y$,

(iv) $T^{k+1}\left(w_{0}\right) \downarrow T(w)$.

Then, $v=T(v)$ and for any other fuxed point $u \in\left[v_{0}, w_{0}\right]$ of $T, v \leq u$. (Then, $w=T(w)$ and for any other fixed point $u \in\left[v_{0}, w_{0}\right]$ of $T, u \leq w$. )

Moreover, if $T$ possesses both properties (i) and (i)', then the sequences $\left\{T^{k}\left(v_{0}\right)\right\},\left\{T^{k}\left(w_{0}\right)\right\}$ are well defined and if, further, $T$ has the properties (iii), (iii)' and (iv), (iv)' then

$$
\begin{gathered}
v_{0} \leq T\left(v_{0}\right) \leq \cdots \leq T^{k}\left(v_{0}\right) \leq \cdots \leq v \leq w \\
\leq \cdots \leq T^{k}\left(w_{0}\right) \leq \cdots \leq T\left(w_{0}\right) \leq w_{0}
\end{gathered}
$$

and $v=T(v), w=T(w)$, and also any other fixed point $u \in\left[v_{0}, w_{0}\right]$ satisfies $v_{0} \leq u \leq w_{0}$.

In the space $C^{(p, q)}\left[I_{a b}, R\right], 0 \leq p+q<n+m$, we introduce a partial ordering as follows: for $v, w \in C^{(p, q)}\left[I_{a b}, R\right]$, we say $v \leq w$ if and only if $\partial^{\mu+\nu} v / \partial x^{\mu} \partial y^{\nu} \leq \partial^{\mu+\nu} w / \partial x^{\mu} \partial y^{\nu} ; 0 \leq \mu \leq n, 0 \leq \nu \leq m, \mu+\nu<n+m$ on 
$I_{a b}$. In this space we also define for norm,

$$
\|u\|=\max _{\substack{0 \leq \mu \leq n \\ 0 \leq \nu \leq m \\ \mu+\nu<n+m}}\left\{\sup _{I_{a b}}\left|\frac{\partial^{\mu+\nu} u}{\partial x^{\mu} \partial y^{\nu}}\right|\right\} .
$$

THEOREM 5.2. Suppose that

(i) $v_{0}, w_{0} \in C^{(n, m)}\left[I_{a b}, R\right]$ are such that $v_{0} \leq w_{0}$ in the partial ordering of $C^{(p, q)}\left[I_{a b}, R\right]$,

(ii) $\partial^{n+m} v_{0} / \partial x^{n} \partial y^{m} \leq f\left(x, y,\left\langle v_{0}\right\rangle\right), \partial^{n+m} w_{0} / \partial x^{n} \partial y^{m} \geq f\left(x, y,\left\langle w_{0}\right\rangle\right)$,

(iii) for fixed nonnegative constants $M_{1}$ and $M_{2}, \varphi_{v_{0}} \leq \varphi \leq \varphi_{w_{0}}$ in the partial ordering of $C^{(p, q)}\left[I_{a b}, R\right]$, where the function $\varphi(x, y)$ is defined in (4.3), $\varphi_{v_{0}}(x, y)$ is same as $\varphi(x, y)$ replacing $\alpha_{i}(x)$ by $\partial^{j} v_{0}(x, 0) / \partial x^{j}$ and $\beta_{i}(y)$ by $\partial^{i} v_{0}(0, y) / \partial y^{i}$, and $\varphi_{w_{0}}(x, y)$ is same as $\varphi(x, y)$ replacing $\alpha_{j}(x)$ by $\partial^{j} w_{0}(x, 0) / \partial x^{j}$ and $\beta_{i}(y)$ by $\partial^{i} w_{0}(0, y) / \partial y^{i}$,

(iv)

$$
\begin{aligned}
& f\left(x, y, u_{0,0}, u_{1,0}, \ldots, u_{n-1, m}\right)-f\left(x, y, \bar{u}_{0,0}, \bar{u}_{1,0}, \ldots, \bar{u}_{n-1, m}\right) \\
& \geq-\sum_{i=0}^{n} \sum_{j=0}^{m}\left(\begin{array}{c}
n \\
i
\end{array}\right)\left(\begin{array}{c}
m \\
j
\end{array}\right)\left(-M_{1}\right)^{n-i}\left(-M_{2}\right)^{m-j}\left(u_{i, k}-\bar{u}_{i, k}\right)
\end{aligned}
$$

wherever $\partial^{\mu+\nu} v_{0} / \partial x^{\mu} \partial y^{\nu} \leq \bar{u}_{\mu, \nu} \leq u_{\mu, \nu} \leq \partial^{\mu+\nu} w_{0} / \partial x^{\mu} \partial y^{\nu}: 0 \leq \mu \leq n$, $0 \leq \nu \leq m, \mu+\nu<n+m$ on $I_{a b}$.

Then, the sequences $\left\{v_{k}\right\},\left\{w_{k}\right\}$ where the functions $v_{k+1}(x, y), w_{k+1}(x, y)$ are (uniquely) generated by the corresponding scheme (5.1)-(5.3) by taking $u_{0}(x, y)$ as $v_{0}(x, y)$ and $w_{0}(x, y)$ respectively, are well defined and $\left\{v_{k}\right\}$ converges to an element $v \in C^{(p, q)}\left[I_{a b}, R\right],\left\{w_{k}\right\}$ converges to an element $w \in$ $C^{(p, q)}\left[I_{a b}, R\right]$ (the convergence being in the norm of $\left.C^{(p, q)}\left[I_{a b}, R\right]\right)$. Further, in the partial ordering of $C^{(p, q)}\left[I_{a b}, R\right]$,

$$
v_{0} \leq v_{1} \leq \cdots \leq v_{k} \leq \cdots \leq v \leq w \leq \cdots \leq w_{k} \leq \cdots \leq w_{1} \leq w_{0},
$$

$v(x, y)$ and $w(x, y)$ are the solutions of the initial value problem (1.1)-(1.4) and each solution $u(x, y)$ of this problem which is such that $u \in\left[v_{0}, w_{0}\right]$ satisfies $v \leq u \leq w$.

Proof. For any $\eta \in C^{(p, q)}\left[I_{a b}, R\right]$ such that $v_{0} \leq \eta \leq w_{0}$ in the partial ordering of $C^{(p, q)}\left[I_{a b}, R\right]$, consider the initial value problem (4.1), (1.2)-(1.4), where

$$
\begin{aligned}
h(x, y)= & f(x, y,\langle\eta\rangle) \\
& +\sum_{i=0}^{n} \sum_{j=0}^{m}\left(\begin{array}{c}
n \\
i
\end{array}\right)\left(\begin{array}{c}
m \\
j
\end{array}\right)\left(-M_{1}\right)^{n-i}\left(-M_{2}\right)^{m-j} \frac{\partial^{i+j} \eta}{\partial x^{i} \partial y^{j}}
\end{aligned}
$$


It is obvious that this initial value problem has a unique solution $u(x, y)$ on $I_{a b}$. On the interval $\left[v_{0}, w_{0}\right]$ we define a mapping $T$ by $T \eta=u$.

First, we shall show that this operator $T$ is isotone. For this, let $\eta_{1}, \eta_{2} \in$ $C^{(p, q)}\left[I_{a b}, R\right]$ be such that $\eta_{1} \leq \eta_{2}$. Let $u_{1}=T \eta_{1}$ and $u_{2}=T \eta_{2}$. Then $\bar{u}=u_{2}-u_{1}$ satisfies the differential equation

$$
\begin{aligned}
\frac{\partial^{n+m} \bar{u}}{\partial x^{n} \partial y^{m}}= & -\sum_{i=0}^{n} \sum_{j=0}^{m}\left(\begin{array}{c}
n \\
i
\end{array}\right)\left(\begin{array}{c}
m \\
j
\end{array}\right)\left(-M_{1}\right)^{n-i}\left(-M_{2}\right)^{m-j} \frac{\partial^{i+j} \bar{u}}{\partial x^{i} \partial y^{j}} \\
& +f\left(x, y,\left\langle\eta_{2}\right\rangle\right)-f\left(x, y,\left\langle\eta_{1}\right\rangle\right) \\
& +\sum_{i=0}^{n} \sum_{j=0}^{m}\left(\begin{array}{c}
n \\
i
\end{array}\right)\left(\begin{array}{c}
m \\
j
\end{array}\right)\left(-M_{1}\right)^{n-i}\left(-M_{2}\right)^{m-j} \frac{\partial^{i+j}\left(\eta_{2}-\eta_{1}\right)}{\partial x^{i} \partial y^{j}}
\end{aligned}
$$

which from the hypothesis (iv) implies that

$$
\frac{\partial^{n+m} \bar{u}}{\partial x^{n} \partial y^{m}} \geq-\sum_{i=0}^{n} \sum_{j=0}^{m}\left(\begin{array}{c}
n \\
i
\end{array}\right)\left(\begin{array}{c}
m \\
j
\end{array}\right)\left(-M_{1}\right)^{n-i}\left(-M_{2}\right)^{m-j} \frac{\partial^{i+j} \bar{u}}{\partial x^{i} \partial y^{j}} .
$$

Further, since $u_{1}$ and $u_{2}$ satisfy the same initial conditions (1.2)-(1.4), it follows that $\partial^{\mu+\nu} \varphi_{a} / \partial x^{\mu} \partial y^{\nu}=0 ; 0 \leq \mu \leq n, 0 \leq \nu \leq m, \mu+\nu<n+m$ on $I_{a b}$. Thus, Lemma 4.2 is applicable, and as a consequence we find that $u_{1} \leq u_{2}$, or equivalently, $T \eta_{1} \leq T \eta_{2}$ in the partial ordering of $C^{(p, q)}\left[I_{a b}, R\right]$. Thus, the operator $T$ is isotone.

Next, we shall show that $v_{0} \leq T v_{0}$ in the partial ordering of $C^{(p, q)}\left[I_{a b}, R\right]$. For this, $T v_{0}=v_{1}$ satisfies the differential equation

$$
\begin{aligned}
\frac{\partial^{n+m} v_{1}}{\partial x^{n} \partial y^{m}}= & -\sum_{i=0}^{n} \sum_{j=0}^{m}\left(\begin{array}{c}
n \\
i
\end{array}\right)\left(\begin{array}{c}
m \\
j
\end{array}\right)\left(-M_{1}\right)^{n-i}\left(-M_{2}\right)^{m-j} \frac{\partial^{i+j} v_{1}}{\partial x^{i} \partial y^{j}}+f\left(x, y,\left\langle v_{0}\right\rangle\right) \\
& +\sum_{i=0}^{n} \sum_{j=0}^{m}\left(\begin{array}{c}
n \\
i
\end{array}\right)\left(\begin{array}{c}
m \\
j
\end{array}\right)\left(-M_{1}\right)^{n-i}\left(-M_{2}\right)^{m-j} \frac{\partial^{i+j} v_{0}}{\partial x^{i} \partial y^{j}}
\end{aligned}
$$

which on using the hypothesis (ii) gives the inequality

$$
\frac{\partial^{n+m} \bar{v}}{\partial x^{n} \partial y^{m}} \geq-\sum_{i=0}^{n} \sum_{j=0}^{m}\left(\begin{array}{c}
n \\
i
\end{array}\right)\left(\begin{array}{c}
m \\
j
\end{array}\right)\left(-M_{1}\right)^{n-i}\left(-M_{2}\right)^{m-j} \frac{\partial^{i+j} v}{\partial x^{i} \partial y^{j}}
$$

where $v=v_{1}-v_{0}$. Further, from the hypothesis (iii), we have $\varphi_{v_{0}} \leq \varphi$ in the partial ordering of $C^{(p, q)}\left[I_{a b}, R\right]$, which implies that $\partial^{\mu+\nu} \varphi_{v} / \partial x^{\mu} \partial y^{\nu} \geq 0$; $0 \leq \mu \leq n, 0 \leq \nu \leq m, \mu+\nu<n+m$ on $I_{a b}$. Thus, Lemma 4.2 is once again applicable, and in conclusion we have $v_{0} \leq T v_{0}=v_{1}$ in the partial ordering of $C^{(p, q)}\left[I_{a b}, R\right]$. The proof of the inequality $T w_{0} \leq w_{0}$ is similar. Thus, the conditions (i) and (i) ${ }^{\prime}$ of Lemma 5.1 hold and it proves that the sequences $\left\{T^{k} v_{0}=v_{k}\right\},\left\{T^{k} w_{0}=w_{k}\right\}$ are well defined. 
Thus, the sequences $\left\{\partial^{\mu+\nu} v_{k} / \partial x^{\mu} \partial y^{\nu}\right\}$ are nondecreasing and bounded above by $\partial^{\mu+\nu} w_{0} / \partial x^{\mu} \partial y^{\nu}$, and the sequences $\left\{\partial^{\mu+\nu} w_{k} / \partial x^{\mu} \partial y^{\nu}\right\}$ are nonincreasing and bounded below by $\partial^{\mu+\nu} v_{0} / \partial x^{\mu} \partial y^{\nu} ; 0 \leq \mu \leq n, 0 \leq \nu \leq m$, $\mu+\nu<n+m$ on $I_{a b}$. Hence, in conclusion the sequences

$$
\left\{\frac{\partial^{\mu+\nu} v_{k}}{\partial x^{\mu} \partial y^{\nu}}\right\},\left\{\frac{\partial^{\mu+\nu} w_{k}}{\partial x^{\mu} \partial y^{\nu}}\right\} \quad 0 \leq \mu \leq n, 0 \leq \nu \leq m, \mu+\nu<n+m,
$$

are uniformly bounded on $I_{a b}$. Further, since the functions $v_{k}(x, y), w_{k}(x, y)$ are the solutions of appropriate initial value problems, these sequences are equicontinuous also. Thus, the Arzela-Ascoli theorem is applicable and for each fixed $\mu, \nu$ there exist subsequences of

$$
\left\{\partial^{\mu+\nu} v_{k} / \partial x^{\mu} \partial y^{\nu}\right\} \text { and }\left\{\partial^{\mu+\nu} w_{k} / \partial x^{\mu} \partial y^{\nu}\right\}
$$

which converge uniformly on $I_{a b}$. However, since these sequences are monotonic, we conclude that the whole sequences

$$
\left\{\partial^{\mu+\nu} v_{k} / \partial x^{\mu} \partial y^{\nu}\right\} \text { and }\left\{\partial^{\mu+\nu} w_{k} / \partial x^{\mu} \partial y^{\nu}\right\}
$$

converge uniformly to some $\partial^{\mu+\nu} v / \partial x^{\mu} \partial y^{\nu}$ and $\partial^{\mu+\nu} w / \partial x^{\mu} \partial y^{\nu}$, and $v \leq w$ in the partial ordering of $C^{(p, q)}\left[I_{a b}, R\right]$. Summarizing these arguments, we find that $T^{k} v_{0} \uparrow v$ and $T^{k} w_{0} \downarrow w$.

Finally, the continuity of the operator $T$ implies that $T^{k+1} v_{0}=T\left[T^{k} v_{0}\right] \uparrow$ $T v$ and $T^{k+1} w_{0}=T\left[T^{k} w_{0}\right] \downarrow T w$.

Hence, the conditions of Lemma 5.1 are satisfied and the conclusions of Theorem 5.2 follow.

COROLLARY 5.3. Suppose in addition to the hypotheses of Theorem 5.2 the function $f$ satisfies the Lipschitz condition (2.16). Then $v(x, y)=w(x, y)$ on $I_{a b}$, and consequently, there exists a unique solution of the initial value problem (1.1)-(1.4) in the interval $\left[v_{0}, w_{0}\right]$.

\section{References}

[1] R. P. Agarwal, 'On integrodifferential inequalities in two independent variables', $J$. Math. Anal. Appl. 89 (1982), 581-597.

[2] R. P. Agarwal, 'Inequalities involving partial derivatives', J. Math. Anal. Appl. 89 (1982), 628-638.

[3] R. P. Agarwal, Boundary value problems for higher order differential equations, (World Scientific, Singapore and Philadelphia, 1986).

[4] R. P. Agarwal, 'Existence-uniqueness and iterative methods for higher order hyperbolic partial differential equations', submitted. 
[5] R. P. Agarwal and R. A. Usmani, 'Monotone convergence of iterative methods for right focal point boundary value problems', J. Math. Anal. Appl. (to appear).

[6] A. Bellen, 'Monotone methods for periodic solutions of second order scalar functional differential equations', Numer. Math. 42 (1983), 15-30.

[7] M. Paul Devasahayam, 'Monotone iterative scheme for nonlinear hyperbolic boundary value problem', Applicable Anal. 20 (1985), 49-55.

[8] I. Ju. Kovac, 'Application of the theorem of differential inequalities to the Goursat problem for a linear system of partial differential equations', Differencial' nye Uravnenija 1 (1965), 411-420 (in Russian).

[9] G. S. Ladde, V. Lakshmikantham and A. S. Vatsala, Monotone iterative techniques for nonlinear differential equations, (Pitman Advanced Publishing Program, Boston, London, Melbourne, 1985).

[10] V. Lakshmikantham and S. G. Pandit, "The method of upper, lower solutions and hyperbolic partial differential equations', J. Math. Anal. Appl. 105 (1985), 466-477.

[11] V. Lakshmikantham and S. G. Pandit, 'Periodic solutions of hyperbolic partial differential equations', Comput. Math. Appl. 11 (1985), 249-259.

[12] P. De Lucia, 'Su un problem al contorno per un'equazione di tipo iperbolico di ordine $2 m^{\prime}$, Ricerche Mat. 16 (1967), 264-302.

[13] J. Szarski, Differential inequalities, (Monograf. Mat. 43, Warsaw, 1965).

[14] M. Volpato, 'Sugli elementi uniti di transformazioni funzionali: un problema ai limiti per una classe di equazioni alle derivate parziali di tipo iperbolico', Ann. Univ. Ferrara 2 $(1952 / 53), 95-109$.

[15] W. Walter, Differential and integral inequalities, (Springer-Verlag, New York, 1970).

\author{
Department of Mathematics \\ National University of Singapore \\ 10 Kent Ridge Crescent \\ Singapore 0511
}

